\title{
Study of risk factors associated with febrile urinary tract infection in children
}

\author{
*Pradeep Sivaraj ${ }^{1}$, Subal Pradhan ${ }^{2}$, Siba Shankar Beriha ${ }^{2}$, Rashmi Ranjan Barik ${ }^{1}$, Nani Tago ${ }^{1}$, Leena \\ Das $^{3}$, Anil Kumar Mohanty ${ }^{4}$
}

Sri Lanka Journal of Child Health, 2015; 44(3): 143-146

\begin{abstract}
Background: Urinary tract infection (UTI) is a common bacterial infection in children. Even a single confirmed UTI is serious, due to the potential for recurrent UTI, parenchymal damage, hypertension and renal failure.
\end{abstract}

Objective: To find the risk factors associated with febrile UTI in children aged 1 month to 14 years.

Method: All children from 1 month to 14 years of age with culture proven febrile $\left(\geq 101^{0} \mathrm{~F}\right)$ UTI were included in the study. Further evaluation was done based on Indian Society of Paediatric Nephrology guidelines. Demographic data, clinical features and underlying risk factors of UTI were studied and analysed.

Results: Out of 79 cases of febrile UTI, majority were boys aged more than 5 years. Escherichia coli was the predominant cause of febrile UTI in $73.4 \%$ cases. Among risk factors, improper toilet training practice was present in $73 \%$, recurrent UTI in $23 \%$ and a history of catheterization /instrumentation in $14 \%$ children. Structural anomalies were found only in $6.3 \%$ cases.

Conclusion: Improper toilet training practices was commonest risk factor associated with febrile UTI in children aged 1 month to 14 years in our study.

(Key words: risk factors, febrile urinary tract infection, children)

\section{Introduction}

Urinary tract infection (UTI) is identified by growth of a significant number of organisms of a single species in the urine, in the presence of symptoms ${ }^{1}$. In young children, the only symptom of a UTI may be fever. Infants may feed poorly, vomit, sleep more or show signs of jaundice. In

${ }^{1}$ Resident, ${ }^{2}$ Assistant Professor, ${ }^{3}$ Associate
Professor, ${ }^{4}$ Professor, Department of Paediatrics,
SVPPGIP, SCB Medical College, Cuttack, India
${ }^{*}$ Correspondence: drspdeep@gmail.com

(Received on 15 August 2014: Accepted after revision on 19 September 2014) older children, new onset urinary incontinence may occur $^{2}$. Even a single confirmed UTI should be taken seriously, especially in young children, due to the potential for recurrent UTI, renal parenchymal damage and long term sequelae of hypertension and renal failure ${ }^{1}$.

\section{Objectives}

To find the risk factors associated with febrile UTI in children aged 1 month to 14 years

\section{Method}

A prospective study was conducted in the Paediatric Departments of SVPPGIP (Shishu bhawan) \& SCB Medical Colleges, Cuttack, Odissa, India, from June 2012 to May 2013. All children aged 1 month to 14 years with a history of fever, clinical symptoms of UTI and positive urine culture with significant colony count were included in the study. Children were included after excluding other causes of fever through history and relevant investigations. Patients with pyuria and no evidence of positive urine cultures were excluded. Children with positive urine cultures with suspected contamination i.e. mixed growth of two or more pathogens, were also excluded from the study. Urine for culture was collected by suprapubic aspiration in infants and by midstream clean catch urine or urinary catheterisation samples in children more than 1 year. Their demographic data and risk factors such as improper toilet training practices, history of $(\mathrm{H} / \mathrm{O})$ recurrent $\mathrm{UTI}$, catheterisation/instrumentation, $\mathrm{H} / \mathrm{O}$ previous surgery, $\mathrm{H} / \mathrm{O}$ constipation, tight clothing and retention of urine, phimosis (male), labial adhesion (female), dehydration status, neurological deficits, palpable faecal mass, circumcision (male), post void dibbling, abnormal urinary stream, neurogenic bladder, ano-rectal malformation and myelomeningocele were noted. Further invasive investigations and workup were done based on Indian Society of Paediatric Nephrology (ISPN) guidelines $2011^{1}$.

Signs of a child's toilet learning readiness are as follows ${ }^{3}$ :

- Able to walk to the potty chair (or adapted toilet seat) 
Study of risk factors associated with febrile urinary tract infection in children Sri Lanka Journal of Child Health, 2015; 44(3): 143-146

- Stable while sitting on the potty (or adapted toilet seat)

- Able to remain dry for several hours

- Receptive language skills allow the child to follow simple (one- and two-step) commands

- Expressive language skills permit the child to communicate the need to use the potty (or adapted toilet seat) with words or reproducible gestures

- Desire to please based on positive relationship with caregivers

- Desire for independence, and control of bladder and bowel function

The following were considered improper toilet training practices:

1. Toilet training of the child by the parents before 18 months of age in both sexes.

2. Initiation of toilet training before the symptoms of readiness appeared.

\section{Results}

Of the 79 cases of febrile UTI, 60 were boys with a male: female ratio of $3: 1$. Most children were older than 5 years at the time of presentation. Forty percent of children had urgency and frequency as presenting symptoms. The microorganisms isolated from urine culture in children with febrile UTI are shown in Table 1.
Table 1

Microorganisms isolated from urine culture

\begin{tabular}{|l|c|}
\hline \multicolumn{1}{|c|}{ Microorganism } & Number (\%) \\
\hline Escherichia coli & $58(73.4)$ \\
\hline Enterococcus & $14(17.7)$ \\
\hline Citrobacter & $06(07.6)$ \\
\hline Candida & $01(01.3)$ \\
\hline
\end{tabular}

The major risk factors associated with febrile UTI are shown in Table 2.

Table 2

Major risk factors associated with febrile UTI

\begin{tabular}{|l|c|}
\hline \multicolumn{1}{|c|}{ Risk Factor } & Number (\%) \\
\hline Improper toilet training & $58(73.4)$ \\
\hline Phimosis & $20(25.3)$ \\
\hline Previous UTI & $18(22.8)$ \\
\hline $\begin{array}{l}\text { H/O instrumentation/ } \\
\text { catheterisation }\end{array}$ & $11(13.9)$ \\
\hline H/O constipation & $10(12.7)$ \\
\hline Tight clothing & $09(11.4)$ \\
\hline Structural anomalies & $05(06.3)$ \\
\hline
\end{tabular}

Other risk factors such as dehydration status, neurological deficits, palpable faecal masses, labial adhesions (female), circumcision (male), post void dibbling, abnormal urinary stream, neurogenic bladder, H/O previous surgery, ano-rectal malformation and myelomeningocele were insignificant in our study. Renal scars were detected only in $2(2.5 \%)$ cases.

Table 3

Recurrent UTI, toilet training and structural anomalies in children based on the age

\begin{tabular}{|l|c|c|c|}
\hline \multicolumn{1}{|c|}{ Risk Factor } & $<\mathbf{1}$ year $(\mathbf{n = 1 2})$ & $\mathbf{1 - 5}$ years $(\mathbf{n}=\mathbf{2 9})$ & $>\mathbf{5}$ years $(\mathbf{n}=\mathbf{3 8})$ \\
\hline Recurrent UTI & 02 & 06 & 10 \\
\hline Proper toilet training & 00 & 05 & 16 \\
\hline Structural anomalies & 03 & 01 & 01 \\
\hline
\end{tabular}

Recurrent UTI was more common among girls $(46 \%)$ than boys $(25 \%)$. Proper toilet training was found in less than $50 \%$ of the age group more than 5 years. Structural anomalies were more common in infants. (Table 3)

\section{Discussion}

In most studies incidence of UTI is more in females than males. For example, in Mingin et al study, 21 were boys and 57 were girls ${ }^{4}$. The classical features of UTI are absent in young children, who often present with few signs or symptoms other than fever ${ }^{5}$. Our study was done in only symptomatic febrile cases in which males were more common than females. The most common presentation was urgency and frequency around $40 \%$. In the Mingin et al study, more than $50 \%$ of cases (13 boys and
28 girls) were under 1 year of age 4 . In our study only $15 \%$ of cases were under 1 year of age, $48 \%$ being more than 5 years.

In the Mingin et al study, none of the boys and girls younger than 1 year old, all $7(100 \%)$ of the 2 to 5 year old boys, $14(82 \%)$ of the 17 girls 2 to 5 years old and all $12(100 \%)$ of the girls older than 5 years were toilet trained at the time of the initial infection. In our study $73 \%$ of the children, irrespective of age and sex, were not properly toilet trained. Most of the parents were unaware of toilet training and its importance due to their low level of education. Similar to Mingin et al study, in our study about $83 \%$ and $58 \%$ were not toilet trained in case of 1-5 year and more than 5 year age groups respectively. All children less than 1 year were not toilet trained. 
Toilet training helps children learn to completely empty their bladder, an ability that reduces the risk of infection ${ }^{6}$. Both the American Academy of Paediatrics and the Canadian Paediatric Society's recommendations for toilet training suggest that children should not be forced into toilet training until they are behaviourally, emotionally and developmentally ready. The training should begin after 18 months of age using a potty-chair and parents should assess readiness signs that show child's interest in toilet training. A study was conducted in 2002 to evaluate the age at which potty training readiness signs were attained. It was found that girls started potty training at an average age of 23 months and boys at an average age of 25 months. The length of time to potty train two year olds ranged from 6.9-14.6 months ${ }^{7}$. Most children in western countries achieve bladder and bowel control between 24 and 48 months of age. Girls tend to achieve this control at a slightly younger age than boys ${ }^{8}$.

In the US, common methods include guidelines of the American Academy of Paediatrics and intensive toilet training method by Azrin and Foxx. However, in developing nations, methods commonly employed are operant conditioning, assisted infant toilet training and elimination communication ${ }^{9}$. To help facilitate the toilet learning process, physicians should inform parents about the 'child-oriented' approach before the process starts, and they should be prepared to offer anticipatory guidance to parents as the child learns toileting skills. In western culture, a child-centred approach, where the timing and method of toilet learning is individualized as much as possible, is recommended ${ }^{8}$.

In the Mingin et al study, out of 78 children, $32 \%$ ( $45 \%$ of the girls and $14 \%$ of the boys) had recurrent $\mathrm{UTI}^{3}$. Recurrences are common, with nearly half the children getting a second infection within a year. After the first episode, expect a recurrence rate of $30 \%$. This rate will double for each subsequent infection ${ }^{5}$. The patient's age at the time of first infection was not predictive of recurrence. In our study recurrence rate was $23 \%$ in which girls had high recurrence rate of $46 \%$ and boys had a recurrence rate of only $25 \%$. Out of 60 boys, 58 boys were uncircumcised; only 2 children were circumcised due to recurrent UTI.

An Arab study showed E. coli was the most commonly isolated pathogen $(60 \%)$, followed by other Gram-negative organisms like Klebsiella $(12 \%)$ and Enterococcus faecalis $(10 \%)^{10}$. In our study, E. coli was the commonest organism isolated from urine culture, which is similar to other studies. Renal ultrasound showed minor abnormalities in $24 / 149 \quad(16 \%)$, vesicourethrography demonstrated vesicoureteral reflux in $38 / 127(30 \%)$ patients and DMSA revealed renal scars in $7 / 28(25 \%)$ children with vesicoureteral reflux $(\mathrm{VUR})^{10}$. In our study there were only $5(6.5 \%)$ structural anomalies (primary VUR in 3 cases, horse shoe kidney in one case and posterior urethral valves in 1 case). Only 12 children were less than one year of age and of that three $(25 \%)$ had structural anomalies. Febrile urinary tract infections (UTI) have long been considered amongst the most common serious bacterial infections in childhood with renal scarring a frequent outcome ${ }^{12}$. In our study renal scarring was found in only $2(2.2 \%)$ cases.

\section{Conclusions}

Improper toilet training practices was the commonest risk factor associated with febrile UTI in children aged 1 month to 14 years in our study, occurring in $73.4 \%$ cases.

\section{References}

1. Indian Society of Pediatric Nephrology. Revised statement on management of urinary tract infections. Indian Pediatrics 2011; 48: 709-717

PMid: 21992903

2. Bhat RG, Katy TA, Place FC. Paediatric urinary tract infections. Emergency Medicine Clinics of North America 2011; 29(3): 637-53.

http://dx.doi.org/10.1016/j.emc.2011.04.0 04

PMid: 21782079

3. Toilet learning: Anticipatory guidance with a child-oriented approach. Paediatrics and Child Health 2000; 5(6): 333-5. PMid: 20177551 PMCid: PMC2819951

4. Mingin GC, Hinds A, Nguyen HT, Baskin LS. Children with a febrile urinary tract infection and a negative radiologic workup: factors predictive of recurrence. Urology 2004; 63(3): 562-5.

http://dx.doi.org/10.1016/j.urology.2003.1 0.055

PMid: 15028458

5. Schlager TA, Loher JA. Urinary tract infection in outpatient febrile infants and children younger than 5 years of age. Pediatric Annals 1993; 22: 505-9. http://dx.doi.org/10.3928/0090-448119930801-11 
Study of risk factors associated with febrile urinary tract infection in children Sri Lanka Journal of Child Health, 2015; 44(3): 143-146

6. Nijman JM, Butler R, Gool JV. Conservative management of urinary incontinence in childhood Journal of Paediatric Urology 2000:513-51

7. Potty Training Early. Available from: http://pottytrainingearly.com/pottytraining -resources

8. Toilet learning: Anticipatory guidance with a child-oriented approach Paediatrics and Child Health 2000; 5(6): 333-5. PMid: 20177551 PMCid: PMC2819951

9. Azrin NH, Foxx RM. A rapid method of toilet training for the institutionalized retarded. Journal of Applied Behaviour Analysis 1971; 4(2): 89-99.

http://dx.doi.org/10.1901/jaba.1971.4-89

PMCid: PMC1310676
10. Ali EMA, Osmanb AH. Acute urinary tract infections in children in Khartoum State: Pathogens, antimicrobial susceptibility and associated risk factors. Arab Journal of Nephrology and Transplantation 2009; 2(2):11-5.

11. Wald E: Urinary tract infections in infants and children: a comprehensive overview. Current Opinion in Pediatrics 2004; 16(1):85-8.

http://dx.doi.org/10.1097/00008480200402000-00016

12. Sakran W, Miron D, Halevy R, Colodner $\mathrm{R}$, Smolkin V, Koren A. Community acquired urinary tract infection among hospitalized children in northern Israel: pathogens, susceptibility patterns and urinary tract anomalies. Harefuah 2003; 142(4):249-52.

PMid: 12754871 